\title{
SYSTEMS WITH FAILURE-DEPENDENT LIFETIMES OF COMPONENTS
}

\author{
M. BURKSCHAT,* Otto-von-Guericke University Magdeburg
}

\begin{abstract}
A model for describing the lifetimes of coherent systems, where the failures of components may have an impact on the lifetimes of the remaining components, is proposed. The model is motivated by the definition of sequential order statistics (cf. Kamps (1995)). Sequential order statistics describe the successive failure times in a sequential $k$-out-of- $n$ system, where the distribution of the remaining components' lifetimes is allowed to change after every failure of a component. In the present paper, general component lifetimes which can be influenced by failures are considered. The ordered failure times of these components can be used to extend the concept of sequential order statistics. In particular, a definition of sequential order statistics based on exchangeable components is proposed. By utilizing the system signature (cf. Samaniego (2007)), the distribution of the lifetime of a coherent system with failure-dependent exchangeable component lifetimes is shown to be given by a mixture of the distributions of sequential order statistics. Furthermore, some results on the joint distribution of sequential order statistics based on exchangeable components are given.
\end{abstract}

Keywords: Coherent system; $k$-out-of- $n$ system; sequential order statistics; exchangeable lifetimes; signature

2000 Mathematics Subject Classification: Primary 60E05; 62E10

\section{Introduction}

In reliability theory, the notion of a coherent system is of great importance for modeling technical systems. Let $X_{1}, \ldots, X_{n}$ denote the lifetimes of the components in a coherent system. Then, the lifetime of the system is given by the random variable $T=\phi\left(X_{1}, \ldots, X_{n}\right)$, where $\phi$ denotes a coherent life function which describes the structure of the system (cf. Barlow and Proschan (1975, p. 12) or Esary and Marshall (1970)). In many situations, the distribution of the system lifetime $T$ can be conveniently expressed in terms of the signature of the system (see Samaniego (2007)). Then, the distribution of $T$ is a mixture of the marginal distributions of the ordered failure times $X_{1: n}, \ldots, X_{n: n}$ of the components, where the weights of this mixture are given by the system signature. The concept of the signature has been introduced for independent and identically distributed (i.i.d.) component lifetimes (see Samaniego (1985)) and extended to exchangeable components (see Kochar et al. (1999), Navarro et al. (2005), Navarro and Eryilmaz (2007), and Navarro et al. (2008c)). Further references for various properties of the Samaniego signature, related concepts, and applications are, for instance, Boland et al. (2003), Boland and Samaniego (2004b), Navarro and Shaked (2006), Khaledi and Shaked (2007), Navarro and Eryilmaz (2007), Navarro et al. (2007a), (2007b), (2008a), (2008b), and Navarro and Hernandez (2008).

Received 15 June 2009; revision received 24 July 2009.

* Postal address: Institute of Mathematical Stochastics, Otto-von-Guericke University Magdeburg, D-39016 Magdeburg, Germany. Email address: marco.burkschat@ovgu.de 
In this paper, a general model for describing the lifetimes of coherent systems, where the failures of components may have an impact on the lifetimes of remaining components, is given. Such a model is adequate if failures lead to an increased stress on surviving units or even cause damages to them. In order to describe these situations, in Kamps (1995) a corresponding model was introduced for $k$-out-of- $n$ systems with i.i.d. component lifetimes. A $k$-out-of- $n: F$ system is a particular coherent system consisting of $n$ components that fails if and only if at least $k$ of the components fail. If the components' lifetimes are described by the random variables $X_{1}, \ldots, X_{n}$, then the lifetime of such a system is given by the $k$ th order statistic $X_{k: n}$. In order to take into account possible effects on component lifetimes after failures, Kamps (1995) defined a generalization of (ordinary) order statistics of i.i.d. random variables, the sequential order statistics. The model of sequential order statistics serves to describe the successive failure times in a sequential $k$-out-of- $n$ system, where the distribution of the remaining components' lifetimes is allowed to change after every failure of a component. More details about this model can be found in Kamps (1995), Cramer and Kamps (2001b), and Cramer (2006b). Further aspects of sequential order statistics have been studied, for instance, in Cramer and Kamps (1996), (1998), Cramer (2001), Cramer and Kamps (2001a), (2001b), (2003), Beutner et al. (2007), Revathy and Chandrasekar (2007), Zhuang and $\mathrm{Hu}$ (2007), Balakrishnan et al. (2008), and Beutner (2008).

The general model treated in this paper will yield an extension of sequential order statistics in two directions. First, the concept of sequential order statistics can be extended to exchangeable random variables, that is, the i.i.d. assumption in sequential $k$-out-of- $n$ systems is weakened. Moreover, the underlying idea is considered not only for the restricted setting of $k$-out-of- $n$ systems, but it is also transferred to more general coherent systems. In fact, the presented approach in principal applies to coherent systems with non-independent and not identically distributed components.

The paper is organized as follows. In Section 2, a model for a system with component lifetimes which can be influenced by failures is defined. The relation between these failuredependent component lifetimes and the successive failure times in the system is given. Given an additional exchangeability assumption, we propose to use the ordered failure times in the model to extend the definition of sequential order statistics to exchangeable components. In Section 3, the lifetime of a system with failure-dependent components is considered. In particular, the approach of using sequential order statistics for describing the lifetime of a $k$-out-of- $n$ system with failure-dependent component lifetimes is transferred to lifetimes of general coherent systems. This is done by deriving a signature-based representation of the distribution of the system lifetime in terms of the distributions of sequential order statistics. Finally, in Section 4, some results on the distribution theory of sequential order statistics based on exchangeable components are given. A particular case of an exchangeable distribution resulting from mixing conditional i.i.d. exponential distributed random variables is studied in detail. The section closes with some illustrative examples.

\section{Ordered failure times of components}

In this section a general scheme of describing the successive failure times of components in a system is derived. After every failure, the lifetimes of the remaining components can be modified. To illustrate this idea, we consider the following example.

Example 2.1. Let $X_{*}^{(1)}$ and $X_{*}^{(2)}$ denote the first and the second failure times, respectively, of the components in a two-component parallel system. In particular, $X_{*}^{(2)}$ denotes the lifetime of 
the complete system. At first, the lifetimes of the two components in the system are assumed to be given by

$$
Y_{1}^{(1)}=\frac{X_{1}}{\alpha_{1} V}, \quad Y_{2}^{(1)}=\frac{X_{2}}{\alpha_{1} V}, \quad \text { with } \alpha_{1}>0,
$$

where $X_{1}$ and $X_{2}$ are independent random variables that follow a standard exponential distribution with mean 1 , and $V$ is a random variable independent of $\left(X_{1}, X_{2}\right)$ which is distributed according to a gamma distribution with shape parameter $a>0$ and scale parameter $b>0$.

Here the random variables $X_{1} / \alpha_{1}$ and $X_{2} / \alpha_{1}$ can be interpreted as the single lifetimes of two identical components. The random variable $V$ represents a random influence on the two components caused by the usage in the system. Related models of components sharing a common environment have been considered in, e.g. Lindley and Singpurwalla (1986), Garren and Richards (1998), and Gupta (2002). Observe that the resulting lifetimes $Y_{1}^{(1)}$ and $Y_{2}^{(1)}$ are exchangeable, that is, the distributions of $\left(Y_{1}^{(1)}, Y_{2}^{(1)}\right)$ and $\left(Y_{2}^{(1)}, Y_{1}^{(1)}\right)$ are identical. Furthermore, for reasons of identifiability, we can assume without loss of generality that $b=1 / a$ for the parameter of the gamma distribution (and, therefore, $\mathrm{E}(V)=1$ ). Due to the assumptions, we obtain the following joint survival function of $\left(Y_{1}^{(1)}, Y_{2}^{(1)}\right)$ :

$$
\begin{aligned}
\mathrm{P}\left(Y_{1}^{(1)}>y_{1}, Y_{2}^{(1)}>y_{2}\right) & =\int_{0}^{\infty} \exp \left[-\alpha_{1} v\left(y_{1}+y_{2}\right)\right] \frac{(a v)^{a-1}}{\Gamma(a)} \mathrm{e}^{-a v} \mathrm{~d} v \\
& =\left(1+\frac{\alpha_{1}\left(y_{1}+y_{2}\right)}{a}\right)^{-a}, \quad y_{1}, y_{2}>0 .
\end{aligned}
$$

Then, the distribution of the first failure time $X_{*}^{(1)}$ in the system is given by $(x>0)$

$$
\mathrm{P}\left(X_{*}^{(1)} \leq x\right)=\mathrm{P}\left(Y_{1: 2}^{(1)} \leq x\right)=1-\mathrm{P}\left(Y_{1}^{(1)}>x, Y_{2}^{(1)}>x\right)=1-\left(1+\frac{2 \alpha_{1} x}{a}\right)^{-a} .
$$

In particular, we obtain the corresponding density function

$$
f^{X_{*}^{(1)}}(x)=2 \alpha_{1}\left(1+\frac{2 \alpha_{1} x}{a}\right)^{-(a+1)}, \quad x>0 .
$$

After the failure of the first component, it is assumed that the surviving component is exposed to a higher load. This is reflected by replacing the parameter $\alpha_{1}$ in (2.1) with a (typically larger) parameter $\alpha_{2}$, which leads to the new model

$$
Y_{1}^{(2)}=\frac{X_{1}}{\alpha_{2} V}, \quad Y_{2}^{(2)}=\frac{X_{2}}{\alpha_{2} V}, \quad \text { with } \alpha_{2}>0 .
$$

The (conditional) distribution of the second failure time $X_{*}^{(2)}$ is now defined via the conditional distribution of the order statistics $Y_{1: 2}^{(2)}$ and $Y_{2: 2}^{(2)}$ of the new random variables $Y_{1}^{(2)}$ and $Y_{2}^{(2)}$ :

$$
\begin{aligned}
\mathrm{P}\left(X_{*}^{(2)} \leq x_{2} \mid X_{*}^{(1)}=x_{1}\right) & =\mathrm{P}\left(Y_{2: 2}^{(2)} \leq x_{2} \mid Y_{1: 2}^{(2)}=x_{1}\right) \\
& =\int_{0}^{x_{2}} f^{Y_{2: 2}^{(2)} \mid Y_{1: 2}^{(2)}}\left(z \mid x_{1}\right) \mathrm{d} z, \quad x_{1} \leq x_{2} .
\end{aligned}
$$

We obtain the representation

$$
\begin{aligned}
f^{X_{*}^{(2)} \mid X_{*}^{(1)}}\left(x_{2} \mid x_{1}\right) & =f^{Y_{2: 2}^{(2)} \mid Y_{1: 2}^{(2)}\left(x_{2} \mid x_{1}\right)} \\
& =\alpha_{2} \frac{a+1}{a} \frac{\left(1+\alpha_{2}\left(x_{1}+x_{2}\right) / a\right)^{-(a+2)}}{\left(1+\alpha_{2} x_{1} / a\right)^{-(a+1)}}, \quad x_{1} \leq x_{2},
\end{aligned}
$$


because of

$$
\begin{aligned}
& f^{Y_{1: 2}^{(2)}, Y_{2: 2}^{(2)}}\left(x_{1}, x_{2}\right)=2 a(a+1)\left(\frac{\alpha_{2}}{a}\right)^{2}\left(1+\frac{\alpha_{2}\left(x_{1}+x_{2}\right)}{a}\right)^{-(a+2)}, \quad x_{1} \leq x_{2}, \\
& f^{Y_{1: 2}^{(2)}}\left(x_{1}\right)=2 \alpha_{2}\left(1+\frac{2 \alpha_{2} x_{1}}{a}\right)^{-(a+1)}, \quad x_{1}>0 .
\end{aligned}
$$

Consequently, the joint density of the two failure times in the 2-out-of-2 system is given by (see (2.2) and (2.3))

$$
\begin{aligned}
f^{X_{*}^{(1)}, X_{*}^{(2)}}\left(x_{1}, x_{2}\right) & =f^{X_{*}^{(1)}}\left(x_{1}\right) f^{X_{*}^{(2)} \mid X_{*}^{(1)}}\left(x_{2} \mid x_{1}\right) \\
& =2 \alpha_{1} \alpha_{2} \frac{a+1}{a}\left(1+\frac{2 \alpha_{1} x}{a}\right)^{-(a+1)} \frac{\left(1+\alpha_{2}\left(x_{1}+x_{2}\right) / a\right)^{-(a+2)}}{\left(1+2 \alpha_{2} x_{1} / a\right)^{-(a+1)}}, \quad x_{1} \leq x_{2} .
\end{aligned}
$$

By construction, in this model the influence of a loss of one component is taken into account by switching to another distribution of the underlying component lifetimes after the first failure.

Now, the basic idea presented in the situation of Example 2.1 is developed in a more general context. In the following, let $n \in \mathbb{N}$ and let $\left(Y_{1}^{(r)}, \ldots, Y_{n}^{(r)}\right), 1 \leq r \leq n$, be random vectors with values in $\mathbb{R}^{n}$ that satisfy the following conditions.

(A1) The random vectors $\left(Y_{1}^{(r)}, \ldots, Y_{n}^{(r)}\right), 1 \leq r \leq n$, have the same support.

(A2) The random variables $Y_{1}^{(r)}, \ldots, Y_{n}^{(r)}$ fulfill

$$
\mathrm{P}\left(Y_{i}^{(r)}=Y_{j}^{(r)}\right)=0, \quad i, j \in\{1, \ldots, n\}, i \neq j,
$$

for every $r \in\{1, \ldots, n\}$.

Note that, for fixed $r \in\{1, \ldots, n\}$, the entries of the random vector $\left(Y_{1}^{(r)}, \ldots, Y_{n}^{(r)}\right)$ need not be independent. Moreover, in contrast to Example 2.1, the random variables $Y_{1}^{(r)}, \ldots, Y_{n}^{(r)}$ need not be necessarily exchangeable.

Furthermore, let $Y_{1: n}^{(r)}, \ldots, Y_{n: n}^{(r)}$ denote the order statistics of the random variables $Y_{1}^{(r)}, \ldots$, $Y_{n}^{(r)}, 1 \leq r \leq n$. For the given random variables $Y_{1}^{(r)}, \ldots, Y_{n}^{(r)}$, we consider the antiranks $I_{1}^{(r)}, \ldots, I_{n}^{(r)}$ :

$$
I_{j}^{(r)}=l \quad \Longleftrightarrow \quad Y_{j: n}^{(r)}=\rho_{j: n}\left(Y_{1}^{(r)}, \ldots, Y_{n}^{(r)}\right)=Y_{l}^{(r)},
$$

where

$$
\rho_{j: n}\left(y_{1}, \ldots, y_{n}\right)=y_{j: n}, \quad y_{1}, \ldots, y_{n} \in \mathbb{R},
$$

and $y_{j: n}$ denotes the $j$ th order statistic (i.e. the $j$ th smallest value among the values $y_{1}, \ldots, y_{n}$ ).

Now, we will specify a model for the distribution of successive failure times in a technical system. At first, the distribution of new random vectors

$$
\left(X_{1}^{(r)}, \ldots, X_{n}^{(r)}\right), \quad 1 \leq r \leq n
$$

is defined in the following way. For $j \in\{1, \ldots, n\}$, let

$$
\begin{aligned}
& \mathrm{P}\left(X_{1}^{(j)} \leq y_{1}, \ldots, X_{n}^{(j)} \leq y_{n} \mid X_{* *}^{(j-1)}=x_{j-1}, I_{* *}^{(j-1)}=i_{j-1}, \ldots, X_{* *}^{(1)}=x_{1}, I_{* *}^{(1)}=i_{1}\right) \\
& \quad=\mathrm{P}\left(Y_{1}^{(j)} \leq y_{1}, \ldots, Y_{n}^{(j)} \leq y_{n} \mid Y_{j-1: n}^{(j)}=x_{j-1}, I_{j-1}^{(j)}=i_{j-1}, \ldots, Y_{1: n}^{(j)}=x_{1}, I_{1}^{(j)}=i_{1}\right) .
\end{aligned}
$$


(Interpretation. Conditioned on the past, $X_{1}^{(j)}, \ldots, X_{n}^{(j)}$ describe the lifetimes of the components in a system with $n$ units before the $j$ th failure has occurred, i.e. $X_{1}^{(j)}$ describes component $1, X_{2}^{(j)}$ describes component 2 , and so on, if the components are labeled with the numbers $1, \ldots, n$. The conditional distribution of $X_{1}^{(j)}, \ldots, X_{n}^{(j)}$ after the $(j-1)$ th and before the $j$ th failure is modeled with the conditional distribution of $Y_{1}^{(j)}, \ldots, Y_{n}^{(j)}$. Note that, due to the conditioning on the past and assumption (A2), after the $(j-1)$ th failure exactly $j-1$ variables of $X_{1}^{(j)}, \ldots, X_{n}^{(j)}$ are determined.) Here

$$
X_{* *}^{(j)}:=\rho_{j: n}\left(X_{1}^{(j)}, \ldots, X_{n}^{(j)}\right)=X_{j: n}^{(j)}
$$

(that is, $X_{* *}^{(j)}$ equals the $j$ th failure time of a unit in the system) and

$$
I_{* *}^{(j)}=l \quad \Longleftrightarrow \quad \rho_{j: n}\left(X_{1}^{(j)}, \ldots, X_{n}^{(j)}\right)=X_{l}^{(j)}
$$

(that is, $I_{* *}^{(j)}$ equals the number of the $j$ th failed unit, i.e. the failure time $X_{* *}^{(j)}$ corresponds to the component with number $\left.I_{* *}^{(j)}\right)$. Note that, due to assumption (A2), the values of $I_{* *}^{(1)}, \ldots, I_{* *}^{(n)}$ are pairwise different and they coincide with the numbers $1, \ldots, n$.

The failure times associated to the different components in the system are finally given by the random variables $X_{1}^{(n)}, \ldots, X_{n}^{(n)}$ from the last stage of the above scheme, that is, $X_{1}^{(n)}$ describes the lifetime of component $1, X_{2}^{(n)}$ describes the lifetime of component 2, and so on. Due to their construction, we will call the random variables $X_{1}^{(n)}, \ldots, X_{n}^{(n)}$ in the following failuredependent component lifetimes. By utilizing the definition of $X_{1}^{(n)}, \ldots, X_{n}^{(n)}$, it is clear that the corresponding order statistics, $X_{1: n}^{(n)}, \ldots, X_{n: n}^{(n)}$, coincide in distribution with the successive failure times $X_{* *}^{(1)}, \ldots, X_{* *}^{(n)}$ (which have been introduced as particular order statistics in the successive stages), that is, we get the following relation.

Lemma 2.1. Under assumptions (A1) and (A2),

$$
\left(X_{1: n}^{(n)}, \ldots, X_{n: n}^{(n)}\right) \sim\left(X_{* *}^{(1)}, \ldots, X_{* *}^{(n)}\right) .
$$

By going through the lines of the previous stepwise construction we observe that the distribution of $X_{* *}^{(1)}, \ldots, X_{* *}^{(n)}$ is determined by the conditional distributions

$$
\begin{array}{r}
\mathrm{P}\left(X_{* *}^{(j+1)} \leq x_{j+1}, I_{* *}^{(j+1)}=i_{j+1} \mid X_{* *}^{(1)}=x_{1}, I_{* *}^{(1)}=i_{1}, \ldots, X_{* *}^{(j)}=x_{j}, I_{* *}^{(j)}=i_{j}\right) \\
=\mathrm{P}\left(Y_{j+1: n}^{(j+1)} \leq x_{j+1}, I_{j+1}^{(j+1)}=i_{j+1} \mid, Y_{1: n}^{(j+1)}=x_{1}, I_{1}^{(j+1)}=i_{1}, \ldots, Y_{j: n}^{(j+1)}=x_{j},\right. \\
\left.I_{j}^{(j+1)}=i_{j}\right)
\end{array}
$$

and the initial distribution

$$
\mathrm{P}\left(X_{* *}^{(1)} \leq x_{1}, I_{* *}^{(1)}=i_{1}\right)=\mathrm{P}\left(Y_{1: n}^{(1)} \leq x_{1}, I_{1}^{(1)}=i_{1}\right) .
$$

Obviously, the random variables $I_{* *}^{(1)}, \ldots, I_{* *}^{(n)}$ may have a strong influence on the joint distribution of $X_{* *}^{(1)}, \ldots, X_{* *}^{(n)}$.

Similarly, we can define, on the basis of assumptions (A1) and (A2), further random variables by neglecting the values of $I_{* *}^{(1)}, \ldots, I_{* *}^{(n)}$. Let $X_{* 0}^{(1)}, \ldots, X_{* 0}^{(n)}$ have a joint distribution that is specified by the conditional distributions

$$
\begin{aligned}
& \mathrm{P}\left(X_{* \circ}^{(j+1)} \leq x_{j+1} \mid X_{* \circ}^{(1)}=x_{1}, \ldots, X_{* \circ}^{(j)}=x_{j}\right) \\
& \quad=\mathrm{P}\left(Y_{j+1: n}^{(j+1)} \leq x_{j+1} \mid Y_{1: n}^{(j+1)}=x_{1}, \ldots, Y_{j: n}^{(j+1)}=x_{j}\right)
\end{aligned}
$$


and the initial distribution

$$
\mathrm{P}\left(X_{* \circ}^{(1)} \leq x_{1}\right)=\mathrm{P}\left(Y_{1: n}^{(1)} \leq x_{1}\right) .
$$

Whereas in the definition of $X_{* *}^{(1)}, \ldots, X_{* *}^{(n)}$ the 'path' of failed components is taken into account, i.e. the number of every unit that has failed in the past appears in the condition of the conditional distributions, in the definition of $X_{* \circ}^{(1)}, \ldots, X_{* \circ}^{(n)}$ this knowledge about the numbers of failed components is not preserved. Therefore, the joint distributions of $X_{* *}^{(1)}, \ldots, X_{* *}^{(n)}$ and $X_{* \circ}^{(1)}, \ldots, X_{* \circ}^{(n)}$ may be different if the components of the random vectors $\left(Y_{1}^{(r)}, \ldots, Y_{n}^{(r)}\right), 1 \leq$ $r \leq n$, are not identically distributed (see Example 2.2, below). However, the distributions of both random vectors coincide in the important case where the following assumption is satisfied.

(A3) The random variables $Y_{1}^{(r)}, \ldots, Y_{n}^{(r)}$ are exchangeable for every $r \in\{1, \ldots, n\}$.

To prove this result, the following lemma will be used. It can be regarded as a generalization of a well-known result for usual order statistics.

Lemma 2.2. Under assumptions (A1), (A2), and (A3), the random vectors

$$
\left(X_{* *}^{(1)}, \ldots, X_{* *}^{(n)}\right) \text { and }\left(I_{* *}^{(1)}, \ldots, I_{* *}^{(n)}\right)
$$

are independent, and

$$
\mathrm{P}\left(I_{* *}^{(1)}=i_{1}, \ldots, I_{* *}^{(n)}=i_{n}\right)=\frac{1}{n !}, \quad\left(i_{1}, \ldots, i_{n}\right) \in \Sigma_{n},
$$

where

$$
\Sigma_{n}:=\left\{\left(r_{1}, \ldots, r_{n}\right) \in\{1, \ldots, n\}^{n} \mid r_{i} \neq r_{j} \text { for } i, j \in\{1, \ldots, n\}, i \neq j\right\} .
$$

Proof. Recall that, due to properties of order statistics from exchangeable random variables (see, e.g. Hájek et al. (1999, p. 37)), for every $r \in\{1, \ldots, n\}$, the random vectors

$$
\left(Y_{1: n}^{(r)}, \ldots, Y_{n: n}^{(r)}\right) \quad \text { and } \quad\left(I_{1}^{(r)}, \ldots, I_{n}^{(r)}\right)
$$

are independent, and

$$
\mathrm{P}\left(I_{1}^{(r)}=i_{1}, \ldots, I_{n}^{(r)}=i_{n}\right)=\frac{1}{n !}, \quad\left(i_{1}, \ldots, i_{n}\right) \in \Sigma_{n} .
$$

Now, in order to prove the lemma, we consider the probabilities

$$
\mathrm{P}\left(X_{* *}^{(1)} \leq x_{1}, \ldots, X_{* *}^{(j)} \leq x_{j}, I_{* *}^{(1)}=i_{1}, \ldots, I_{* *}^{(j)}=i_{j}\right), \quad j \in\{1, \ldots, n\},
$$

and carry out an induction on $j \in\{1, \ldots, n\}$. The case in which $j=n$ proves the assertion. For $j=1$, we obtain

$$
\mathrm{P}\left(X_{* *}^{(1)} \leq x_{1}, I_{* *}^{(1)}=i_{1}\right)=\mathrm{P}\left(Y_{1: n}^{(1)} \leq x_{1}, I_{1}^{(1)}=i_{1}\right)=\mathrm{P}\left(Y_{1: n}^{(1)} \leq x_{1}\right) \mathrm{P}\left(I_{1}^{(1)}=i_{1}\right)
$$

for every $x_{1} \in \mathbb{R}$ and $i_{1} \in\{1, \ldots, n\}$. This yields

$$
\mathrm{P}\left(X_{* *}^{(1)} \leq x_{1}, I_{* *}^{(1)}=i_{1}\right)=\mathrm{P}\left(X_{* *}^{(1)} \leq x_{1}\right) \mathrm{P}\left(I_{* *}^{(1)}=i_{1}\right)
$$


for $j=1$. Now, let the induction assumption hold for $j \geq 1$ with $j<n$. Consider

$$
\begin{gathered}
\mathrm{P}\left(X_{* *}^{(1)} \leq x_{1}, \ldots, X_{* *}^{(j+1)} \leq x_{j+1}, I_{* *}^{(1)}=i_{1}, \ldots, I_{* *}^{(j+1)}=i_{j+1}\right) \\
=\int_{\times_{l=1}^{j}\left(-\infty, x_{l}\right] \times \times_{l=1}^{j}\left\{i_{l}\right\}} \mathrm{P}\left(X_{* *}^{(j+1)} \leq x_{j+1}, I_{* *}^{(j+1)}=i_{j+1} \mid X_{* *}^{(1)}=y_{1}, \ldots, X_{* *}^{(j)}=y_{j},\right. \\
\left.\quad I_{* *}^{(1)}=k_{1}, \ldots, I_{* *}^{(j)}=k_{j}\right) \\
\times \mathrm{d} P^{X_{* *}^{(1)}, \ldots, X_{* *}^{(j)}, I_{* *}^{(1)}, \ldots, I_{* *}^{(j)}}\left(y_{1}, \ldots, y_{j}, k_{1}, \ldots, k_{j}\right) \\
=(\star) .
\end{gathered}
$$

Because, by definition,

$$
\begin{gathered}
\mathrm{P}\left(X_{* *}^{(j+1)} \leq x_{j+1}, I_{* *}^{(j+1)}=i_{j+1} \mid X_{* *}^{(1)}=y_{1}, \ldots, X_{* *}^{(j)}=y_{j}, I_{* *}^{(1)}=k_{1}, \ldots, I_{* *}^{(j)}=k_{j}\right) \\
=\mathrm{P}\left(\rho_{j+1: n}\left(X_{1}^{(j+1)}, \ldots, X_{n}^{(j+1)}\right) \leq x_{j+1}, \rho_{j+1: n}\left(X_{1}^{(j+1)}, \ldots, X_{n}^{(j+1)}\right)=X_{i_{j+1}}^{(j+1)} \mid\right. \\
\left.X_{* *}^{(1)}=y_{1}, \ldots, X_{* *}^{(j)}=y_{j}, I_{* *}^{(1)}=k_{1}, \ldots, I_{* *}^{(j)}=k_{j}\right) \\
=\mathrm{P}\left(\rho_{j+1: n}\left(Y_{1}^{(j+1)}, \ldots, Y_{n}^{(j+1)}\right) \leq x_{j+1}, \rho_{j+1: n}\left(Y_{1}^{(j+1)}, \ldots, Y_{n}^{(j+1)}\right)=Y_{i_{j+1}}^{(j+1)} \mid\right. \\
\left.Y_{1: n}^{(j+1)}=y_{1}, \ldots, Y_{j: n}^{(j+1)}=y_{j}, I_{1}^{(j+1)}=k_{1}, \ldots, I_{j}^{(j+1)}=k_{j}\right) \\
=\mathrm{P}\left(Y_{j+1: n}^{(j+1)} \leq x_{j+1}, I_{j+1}^{(j+1)}=i_{j+1} \mid Y_{1: n}^{(j+1)}=y_{1}, \ldots, Y_{j: n}^{(j+1)}=y_{j},\right. \\
\left.I_{1}^{(j+1)}=k_{1}, \ldots, I_{j}^{(j+1)}=k_{j}\right) .
\end{gathered}
$$

Since $\left(Y_{1: n}^{(j+1)}, \ldots, Y_{n: n}^{(j+1)}\right)$ and $\left(I_{1}^{(j+1)}, \ldots, I_{n}^{(j+1)}\right)$ are independent, we obtain, for the last expression,

$$
\begin{gathered}
\mathrm{P}\left(Y_{j+1: n}^{(j+1)} \leq x_{j+1}, I_{j+1}^{(j+1)}=i_{j+1} \mid Y_{1: n}^{(j+1)}=y_{1}, \ldots, Y_{j: n}^{(j+1)}=y_{j},\right. \\
\left.I_{1}^{(j+1)}=k_{1}, \ldots, I_{j}^{(j+1)}=k_{j}\right) \\
=\mathrm{P}\left(Y_{j+1: n}^{(j+1)} \leq x_{j+1} \mid Y_{1: n}^{(j+1)}=y_{1}, \ldots, Y_{j: n}^{(j+1)}=y_{j}\right) \\
\quad \times \mathrm{P}\left(I_{j+1}^{(j+1)}=i_{j+1} \mid I_{1}^{(j+1)}=k_{1}, \ldots, I_{j}^{(j+1)}=k_{j}\right) .
\end{gathered}
$$

\section{Consequently,}

$$
\begin{gathered}
\mathrm{P}\left(X_{* *}^{(j+1)} \leq x_{j+1}, I_{* *}^{(j+1)}=i_{j+1} \mid X_{* *}^{(1)}=y_{1}, \ldots, X_{* *}^{(j)}=y_{j}, I_{* *}^{(1)}=k_{1}, \ldots, I_{* *}^{(j)}=k_{j}\right) \\
=\mathrm{P}\left(Y_{j+1: n}^{(j+1)} \leq x_{j+1} \mid Y_{1: n}^{(j+1)}=y_{1}, \ldots, Y_{j: n}^{(j+1)}=y_{j}\right) \\
\quad \times \mathrm{P}\left(I_{j+1}^{(j+1)}=i_{j+1} \mid I_{1}^{(j+1)}=k_{1}, \ldots, I_{j}^{(j+1)}=k_{j}\right) .
\end{gathered}
$$

By either summing over $i_{j+1}$ or letting $x_{j+1} \rightarrow \infty$ and using the induction hypothesis, this yields

$$
\begin{aligned}
& \mathrm{P}\left(X_{* *}^{(j+1)} \leq x_{j+1}, I_{* *}^{(j+1)}=i_{j+1} \mid X_{* *}^{(1)}=y_{1}, \ldots, X_{* *}^{(j)}=y_{j}, I_{* *}^{(1)}=k_{1}, \ldots, I_{* *}^{(j)}=k_{j}\right) \\
& =\mathrm{P}\left(X_{* *}^{(j+1)} \leq x_{j+1} \mid X_{* *}^{(1)}=y_{1}, \ldots, X_{* *}^{(j)}=y_{j}\right) \\
& \quad \times \mathrm{P}\left(I_{* *}^{(j+1)}=i_{j+1} \mid I_{* *}^{(1)}=k_{1}, \ldots, I_{* *}^{(j)}=k_{j}\right) .
\end{aligned}
$$


Thus, by again utilizing the induction hypothesis,

$$
\begin{aligned}
&(\star)= \int_{\times_{l=1}^{j}\left(-\infty, x_{l}\right] \times \times_{l=1}^{j}\left\{i_{l}\right\}} \mathrm{P}\left(X_{* *}^{(j+1)} \leq x_{j+1} \mid X_{* *}^{(1)}=y_{1}, \ldots, X_{* *}^{(j)}=y_{j}\right) \\
& \times \mathrm{P}\left(I_{* *}^{(j+1)}=i_{j+1} \mid I_{* *}^{(1)}=k_{1}, \ldots, I_{* *}^{(j)}=k_{j}\right) \\
& \times \mathrm{d}\left(\mathrm{P}^{X_{* *}^{(1)}, \ldots, X_{* *}^{(j)} \otimes \mathrm{P}^{\left.I_{* *}^{(1)}, \ldots, I_{* *}^{(j)}\right)}\left(y_{1}, \ldots, y_{j}, k_{1}, \ldots, k_{j}\right)}\right. \\
&=\int_{\times_{l=1}^{j}\left(-\infty, x_{l}\right]} \mathrm{P}\left(X_{* *}^{(j+1)} \leq x_{j+1} \mid X_{* *}^{(1)}=y_{1}, \ldots, X_{* *}^{(j)}=y_{j}\right) \mathrm{d} P^{X_{* *}^{(1)}, \ldots, X_{* *}^{(j)}}\left(y_{1}, \ldots, y_{j}\right) \\
& \quad \times \int_{\times_{l=1}^{j}\left\{i_{l}\right\}} \mathrm{P}\left(I_{* *}^{(j+1)}=i_{j+1} \mid I_{* *}^{(1)}=k_{1}, \ldots, I_{* *}^{(j)}=k_{j}\right) \mathrm{d} P^{I_{* *}^{(1)}, \ldots, I_{* *}^{(j)}}\left(k_{1}, \ldots, k_{j}\right) \\
&= \mathrm{P}\left(X_{* *}^{(1)} \leq x_{1}, \ldots, X_{* *}^{(j+1)} \leq x_{j+1}\right) \mathrm{P}\left(I_{* *}^{(1)}=i_{1}, \ldots, I_{* *}^{(j+1)}=i_{j+1}\right) .
\end{aligned}
$$

For determining the probabilities, consider

$$
\mathrm{P}\left(I_{* *}^{(1)}=i_{1}, \ldots, I_{* *}^{(n)}=i_{n}\right)=\mathrm{P}\left(I_{* *}^{(1)}=i_{1}\right) \prod_{j=2}^{n} \mathrm{P}\left(I_{* *}^{(j)}=i_{j} \mid I_{* *}^{(j-1)}=i_{j-1}, \ldots, I_{* *}^{(1)}=i_{1}\right) .
$$

Because of

$$
\mathrm{P}\left(I_{* *}^{(j)}=i_{j} \mid I_{* *}^{(j-1)}=i_{j-1}, \ldots, I_{* *}^{(1)}=i_{1}\right)=\mathrm{P}\left(I_{j}^{(j)}=i_{j} \mid I_{j-1}^{(j)}=i_{j-1}, \ldots, I_{1}^{(j)}=i_{1}\right),
$$

the corresponding results for usual order statistics from exchangeable random variables can now be applied. This completes the proof.

Now we show the announced equality in distribution of both random vectors under the exchangeability assumption.

Theorem 2.1. Under assumptions (A1), (A2), and (A3),

$$
\left(X_{* *}^{(1)}, \ldots, X_{* *}^{(n)}\right) \sim\left(X_{* \circ}^{(1)}, \ldots, X_{* \circ}^{(n)}\right) .
$$

Proof. By definition we have

$$
\mathrm{P}\left(X_{* *}^{(1)} \leq x_{1}\right)=\mathrm{P}\left(Y_{1: n}^{(1)} \leq x_{1}\right)=\mathrm{P}\left(X_{* \circ}^{(1)} \leq x_{1}\right), \quad x_{1} \in \mathbb{R} .
$$

Let $\left(i_{1}, \ldots, i_{n}\right) \in \Sigma_{n}$. Then, we obtain, for $r \in\{1, \ldots, n-1\}$,

$$
\begin{aligned}
\mathrm{P} & \left.X_{* *}^{(r+1)} \leq x_{r+1} \mid X_{* *}^{(r)}=x_{r}, \ldots, X_{* *}^{(1)}=x_{1}\right) \\
& =\mathrm{P}\left(X_{* *}^{(r+1)} \leq x_{r+1} \mid X_{* *}^{(r)}=x_{r}, I_{* *}^{(r)}=i_{r}, \ldots, X_{* *}^{(1)}=x_{1}, I_{* *}^{(1)}=i_{1}\right) \quad(\text { by Lemma 2.2) } \\
& =\mathrm{P}\left(Y_{r+1: n}^{(r+1)} \leq x_{r+1} \mid Y_{r: n}^{(r+1)}=x_{r}, I_{r}^{(r+1)}=i_{r}, \ldots, Y_{1: n}^{(r+1)}=x_{1}, I_{1}^{(r+1)}=i_{1}\right) \\
& \quad \text { (by definition) } \\
& =\mathrm{P}\left(Y_{r+1: n}^{(r+1)} \leq x_{r+1} \mid Y_{r: n}^{(r+1)}=x_{r}, \ldots, Y_{1: n}^{(r+1)}=x_{1}\right) \quad \text { (due to assumption (A3)) } \\
& =\mathrm{P}\left(X_{* \circ}^{(r+1)} \leq x_{r+1} \mid X_{* 0}^{(r)}=x_{r}, \ldots, X_{* 0}^{(1)}=x_{1}\right) \quad \text { (by definition). }
\end{aligned}
$$

Thus, the claimed result is proved. 
Since in the situation considered in Theorem 2.1 both types of random variables can be regarded as an extension of the concept of sequential order statistics introduced in Kamps (1995) to exchangeable components, we apply the same notation in this case. Thus, we propose the following definition of sequential order statistics based on exchangeable components.

Definition 2.1. Let $n \in \mathbb{N}$. Let $\left(Y_{1}^{(r)}, \ldots, Y_{n}^{(r)}\right), 1 \leq r \leq n$, be random vectors with values in $\mathbb{R}^{n}$ that satisfy the following conditions.

(A1) The random vectors $\left(Y_{1}^{(r)}, \ldots, Y_{n}^{(r)}\right), 1 \leq r \leq n$, have the same support.

(A2) The random variables $Y_{1}^{(r)}, \ldots, Y_{n}^{(r)}$ fulfill

$$
\mathrm{P}\left(Y_{i}^{(r)}=Y_{j}^{(r)}\right)=0, \quad i, j \in\{1, \ldots, n\}, i \neq j,
$$

for every $r \in\{1, \ldots, n\}$.

(A3) The random variables $Y_{1}^{(r)}, \ldots, Y_{n}^{(r)}$ are exchangeable for every $r \in\{1, \ldots, n\}$.

Let $\left(Y_{1: n}^{(r)}, \ldots, Y_{n: n}^{(r)}\right), 1 \leq r \leq n$, be the random vectors of the corresponding order statistics. Then random variables $X_{*}^{(1)}, \ldots, X_{*}^{(n)}$ are called sequential order statistics based on exchangeable components if their distribution satisfies

$$
\mathrm{P}\left(X_{*}^{(1)} \leq x_{1}\right)=\mathrm{P}\left(Y_{1: n}^{(1)} \leq x_{1}\right), \quad x_{1} \in \mathbb{R},
$$

and

$$
\begin{aligned}
& \mathrm{P}\left(X_{*}^{(r+1)} \leq x_{r+1} \mid X_{*}^{(r)}=x_{r}, \ldots, X_{*}^{(1)}=x_{1}\right) \\
& \quad=\mathrm{P}\left(Y_{r+1: n}^{(r+1)} \leq x_{r+1} \mid Y_{r: n}^{(r+1)}=x_{r}, \ldots, Y_{1: n}^{(r+1)}=x_{1}\right), \quad x_{r+1} \in \mathbb{R},
\end{aligned}
$$

for $\mathrm{P}^{X_{*}^{(r)}}, \ldots, X_{*}^{(1)}$-almost all $\left(x_{r}, \ldots, x_{1}\right) \in \mathbb{R}^{r}$ for every $r \in\{1, \ldots, n-1\}$.

Remark 2.1. The distribution of sequential order statistics coincides with that of usual order statistics based on exchangeable random variables if the distributions of the underlying random vectors $\left(Y_{1}^{(r)}, \ldots, Y_{n}^{(r)}\right), 1 \leq r \leq n$, are identical.

Remark 2.2. The relation to the sequential order statistics based on i.i.d. random variables defined in Kamps (1995) is seen in this way. Let the vectors $\left(Y_{1}^{(r)}, \ldots, Y_{n}^{(r)}\right), 1 \leq r \leq n$, have i.i.d. components with continuous cumulative distribution function (CDF) $F_{r}$. Then,

$$
\mathrm{P}\left(X_{*}^{(1)} \leq x_{1}\right)=\mathrm{P}\left(Y_{1: n}^{(1)} \leq x_{1}\right)=1-\left(1-F_{1}\left(x_{1}\right)\right)^{n}, \quad x_{1} \in \mathbb{R} .
$$

Moreover, due to the Markov property of order statistics from i.i.d. random variables with a continuous distribution and a well-known result for conditional distributions of order statistics (see David and Nagaraja (2003, Theorem 2.5)), we obtain

$$
\begin{aligned}
\mathrm{P}\left(X_{*}^{(r+1)} \leq x_{r+1} \mid X_{*}^{(r)}=x_{r}, \ldots, X_{*}^{(1)}=x_{1}\right) & =\mathrm{P}\left(Y_{r+1: n}^{(r+1)} \leq x_{r+1} \mid Y_{r: n}^{(r+1)}=x_{r}\right) \\
& =1-\left(\frac{1-F_{r+1}\left(x_{r+1}\right)}{1-F_{r+1}\left(x_{r}\right)}\right)^{n-r} .
\end{aligned}
$$

Therefore, $X_{*}^{(1)}, \ldots, X_{*}^{(n)}$ also have the Markov property and their transition probabilities coincide with those of sequential order statistics (cf. Kamps (1995, p. 29)). 
Remark 2.3. Let $Y_{1}^{(r)}, \ldots, Y_{n}^{(r)}$ be i.i.d. random variables that are distributed according to the distribution function

$$
F_{r}=1-(1-F)^{\alpha_{r}}, \quad \alpha_{r}>0,
$$

where $F$ denotes a continuous distribution function. The resulting model of sequential order statistics $X_{*}^{(1)}, \ldots, X_{*}^{(n)}$ has been studied extensively in the literature (we refer the reader to the references in Section 1). In this case, sequential order statistics can be treated as generalized order statistics (see Kamps (1995)) and, therefore, corresponding results apply as well. In the particular situation where $F$ is given by an exponential distribution with location and scale parameters, the joint distribution of $X_{*}^{(1)}, \ldots, X_{*}^{(n)}$ is known to coincide with the distribution of order statistics from a Weinman multivariate exponential distribution (cf. Johnson et al. (2000, pp. 388-391)).

Remark 2.4. The situation given in Example 2.1 fits into the general framework as follows. In fact, there we described the ordered failure times in a two-component parallel system via particular sequential order statistics $X_{*}^{(1)}$ and $X_{*}^{(2)}$. The corresponding component lifetimes are based on random vectors $\left(Y_{1}^{(r)}, Y_{2}^{(r)}\right), r \in\{1,2\}$, with the absolutely continuous exchangeable distributions (with $\alpha_{1}, \alpha_{2}, a>0$ )

$$
\mathrm{P}\left(Y_{1}^{(r)}>y_{1}, Y_{2}^{(r)}>y_{2}\right)=\left(1+\frac{\alpha_{r}\left(y_{1}+y_{2}\right)}{a}\right)^{-a}, \quad y_{1}, y_{2}>0, r \in\{1,2\},
$$

respectively. Therefore, the assumptions of Definition 2.1 are fulfilled.

Of course, given assumption (A3), the distributions of the random variables coincide, i.e.

$$
\left(X_{* *}^{(1)}, \ldots, X_{* *}^{(n)}\right) \sim\left(X_{*}^{(1)}, \ldots, X_{*}^{(n)}\right) \sim\left(X_{* \circ}^{(1)}, \ldots, X_{* \circ}^{(n)}\right) .
$$

If (A3) is dropped, the random variables $X_{* *}^{(1)}, \ldots, X_{* *}^{(n)}$ and $X_{* \circ}^{(1)}, \ldots, X_{* \circ}^{(n)}$ can be regarded as extensions of sequential order statistics based on exchangeable components into two different directions. The random variables $X_{* *}^{(1)}, \ldots, X_{* *}^{(n)}$ can be used to describe failure times in technical systems with nonexchangeable components where failures may affect the lifetimes of remaining intact components. Therefore, these random variables enable the transfer of the idea underlying the concept of sequential order statistics to more general systems. In contrast, $X_{* \circ}^{(1)}, \ldots, X_{* 0}^{(n)}$ represent a direct extension of the formal definition of sequential order statistics to the nonexchangeable case.

We close this section with an example which illustrates that the joint distributions of $X_{* \circ}^{(1)}, \ldots$, $X_{* \circ}^{(n)}$ and $X_{* *}^{(1)}, \ldots, X_{* *}^{(n)}$ may be different.

Example 2.2. Let the random vectors $\left(Y_{1}^{(1)}, Y_{2}^{(1)}\right)$ and $\left(Y_{1}^{(2)}, Y_{2}^{(2)}\right)$ be given, with corresponding density functions

$$
\begin{array}{ll}
f^{Y_{1}^{(1)}, Y_{2}^{(1)}}\left(y_{1}, y_{2}\right)=\lambda_{1} \lambda_{2} \exp \left[-\left(\lambda_{1} y_{1}+\lambda_{2} y_{2}\right)\right], & y_{1}, y_{2}>0, \\
f^{Y_{1}^{(2)}, Y_{2}^{(2)}}\left(y_{1}, y_{2}\right)=\tilde{\lambda}_{1} \tilde{\lambda}_{2} \exp \left[-\left(\tilde{\lambda}_{1} y_{1}+\tilde{\lambda}_{2} y_{2}\right)\right], & y_{1}, y_{2}>0,
\end{array}
$$

with $\lambda_{1}, \lambda_{2}, \tilde{\lambda}_{1}, \tilde{\lambda}_{2}>0$. Thus, $\left(Y_{1}^{(1)}, Y_{2}^{(1)}\right)$ is distributed as two independent random variables that follow exponential distributions with parameters $\lambda_{1}$ and $\lambda_{2}$, respectively. The vector $\left(Y_{1}^{(2)}, Y_{2}^{(2)}\right)$ follows the same law, but with (possibly different) parameters $\widetilde{\lambda}_{1}$ and $\widetilde{\lambda}_{2}$. By following the construction of $\left(X_{1}^{(2)}, X_{2}^{(2)}\right)$ we obtain the corresponding density:

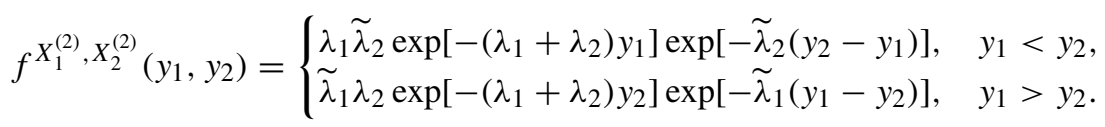


Note that this is Freund's bivariate exponential distribution. Recall that if two random variables $X_{1}$ and $X_{2}$ have a joint density function with respect to the two-dimensional Lebesgue measure, then the density function of the corresponding order statistics is given by

$$
f^{X_{1: 2}, X_{2: 2}}\left(x_{1}, x_{2}\right)=f^{X_{1}, X_{2}}\left(x_{1}, x_{2}\right)+f^{X_{1}, X_{2}}\left(x_{2}, x_{1}\right), \quad x_{1}<x_{2} .
$$

Thus, by applying Lemma 2.1 we obtain the following density function for $\left(X_{* *}^{(1)}, X_{* *}^{(2)}\right)$ :

$$
\begin{aligned}
f^{X_{* *}^{(1)}, X_{* *}^{(2)}}\left(x_{1}, x_{2}\right)= & \exp \left[-\left(\lambda_{1}+\lambda_{2}\right) x_{1}\right] \\
& \times\left(\lambda_{1} \tilde{\lambda}_{2} \exp \left[-\tilde{\lambda}_{2}\left(x_{2}-x_{1}\right)\right]+\tilde{\lambda}_{1} \lambda_{2} \exp \left[-\tilde{\lambda}_{1}\left(x_{2}-x_{1}\right)\right]\right), \quad x_{1}<x_{2} .
\end{aligned}
$$

However, it can be shown that the density function of $\left(X_{* \circ}^{(1)}, X_{* \circ}^{(2)}\right)$ is given by

$$
\begin{aligned}
f^{X_{*}^{(1)}, X_{* o}^{(2)}}\left(x_{1}, x_{2}\right)= & \tilde{\lambda}_{1} \tilde{\lambda}_{2} \frac{\lambda_{1}+\lambda_{2}}{\widetilde{\lambda}_{1}+\tilde{\lambda}_{2}} \exp \left[-\left(\lambda_{1}+\lambda_{2}\right) x_{1}\right] \\
& \times\left(\exp \left[-\tilde{\lambda}_{2}\left(x_{2}-x_{1}\right)\right]+\exp \left[-\tilde{\lambda}_{1}\left(x_{2}-x_{1}\right)\right]\right), \quad x_{1}<x_{2} .
\end{aligned}
$$

Consequently, the distributions of $\left(X_{* *}^{(1)}, X_{* *}^{(2)}\right)$ and $\left(X_{* \circ}^{(1)}, X_{* \circ}^{(2)}\right)$ may be different if the underlying random vectors are not exchangeable. Clearly, in the exchangeable case, i.e. $\lambda_{1}=\lambda_{2}=\lambda$, $\tilde{\lambda}_{1}=\tilde{\lambda}_{2}=\tilde{\lambda}$, both densities are identical (see Theorem 2.1) and we obtain the following density of sequential order statistics:

$$
f^{X_{*}^{(1)}, X_{*}^{(2)}}\left(x_{1}, x_{2}\right)=2 \lambda \tilde{\lambda} \exp \left[-2 \lambda x_{1}\right] \exp \left[-\tilde{\lambda}\left(x_{2}-x_{1}\right)\right], \quad x_{1}<x_{2} .
$$

Note that this is the density function of particular sequential order statistics based on i.i.d. random variables with $\mathrm{CDF} F_{1}$ in the first and $\mathrm{CDF} F_{2}$ in the second stage, where the assumptions $F_{1}=1-(1-F)^{\alpha_{1}}$ and $F_{2}=1-(1-F)^{\alpha_{2}}$ are imposed (cf. Remark 2.3). Here, we arrived at sequential order statistics with underlying standard exponential distribution $F(t)=1-\mathrm{e}^{-t}, t \geq 0$, and the model parameters $\alpha_{1}=\lambda, \alpha_{2}=\widetilde{\lambda}$.

Finally, observe that even in the case $\lambda_{1}=\widetilde{\lambda}_{1}$ and $\lambda_{2}=\widetilde{\lambda}_{2}$, the densities of $\left(X_{* *}^{(1)}, X_{* *}^{(2)}\right)$ and $\left(X_{* 0}^{(1)}, X_{* 0}^{(2)}\right)$ are identical:

$$
\begin{aligned}
f^{X_{* *}^{(1)}, X_{* *}^{(2)}}\left(x_{1}, x_{2}\right) & =\lambda_{1} \lambda_{2} \exp \left[-\left(\lambda_{1}+\lambda_{2}\right) x_{1}\right]\left(\exp \left[-\lambda_{2}\left(x_{2}-x_{1}\right)\right]+\exp \left[-\lambda_{1}\left(x_{2}-x_{1}\right)\right]\right) \\
& =\lambda_{1} \lambda_{2}\left(\exp \left[-\left(\lambda_{1} x_{1}+\lambda_{2} x_{2}\right)\right]+\exp \left[-\left(\lambda_{2} x_{1}+\lambda_{1} x_{2}\right)\right]\right), \quad x_{1}<x_{2} .
\end{aligned}
$$

In this case both random vectors are also not exchangeable, but there is no change in the underlying bivariate distribution after the first failure. Consequently, we obtain usual order statistics from independent, but not identically distributed exponential random variables.

\section{Systems with failure-dependent component lifetimes}

In the last section we developed a model for the increasingly ordered failure times of the components in a system with failure-dependent component lifetimes. Under assumptions (A1) and (A2), the lifetimes of the single components in such a system are given by the random variables $X_{1}^{(n)}, \ldots, X_{n}^{(n)}$. The ordered failure times $X_{* *}^{(1)}, \ldots, X_{* *}^{(n)}$ are distributed like the corresponding order statistics (cf. Lemma 2.1).

In this section we are interested in the lifetime of the system itself. In the following we will consider coherent systems (cf. Barlow and Proschan (1975)). The relation between the lifetime of a coherent system and the lifetimes of its components can be specified in terms of a 
coherent life function $\phi$ (cf. Barlow and Proschan (1975, p. 12) or Esary and Marshall (1970)). Due to the definition of coherent life functions, the lifetime $T^{* *}$ of a coherent system with the failure-dependent component lifetimes $X_{1}^{(n)}, \ldots, X_{n}^{(n)}$ is given by

$$
T^{* *}=\phi\left(X_{1}^{(n)}, \ldots, X_{n}^{(n)}\right) .
$$

Clearly, this relation holds without imposing exchangeability assumptions on the component lifetimes. By additionally assuming (A3), the reliability function of the system lifetime can be represented as a mixture of the reliability functions of sequential order statistics $X_{*}^{(1)}, \ldots, X_{*}^{(n)}$. The weights in the mixture are given by the system signature (cf. Samaniego (2007)).

The signature $s=\left(s_{1}, \ldots, s_{n}\right)$ of a coherent system with coherent life function $\phi$ and exchangeable component lifetimes $X_{1}, \ldots, X_{n}$ such that

$$
\mathrm{P}\left(X_{i}=X_{j}\right)=0, \quad i, j \in\{1, \ldots, n\}, i \neq j,
$$

is given by (this follows from Navarro et al. (2008c, Theorem 3.6))

$$
s_{i}=\mathrm{P}\left(T=X_{i: n}\right), \quad 1 \leq i \leq n,
$$

where $T=\phi\left(X_{1}, \ldots, X_{n}\right)$ denotes the system lifetime. In this case, the signature can also be represented as follows (see Navarro and Eryilmaz (2007), and Navarro et al. (2008c, Theorem 3.2)):

$$
s_{i}=\frac{\#\left\{\sigma \in P_{n} \mid \sigma \in S_{i}\right\}}{n !}, \quad 1 \leq i \leq n,
$$

where $P_{n}$ denotes the set of all permutations of the numbers $1, \ldots, n$ and $S_{i}$ is a set of permutations with

$$
S_{i}=\left\{\sigma \in P_{n} \mid \phi\left(y_{1}, \ldots, y_{n}\right)=y_{i: n} \text { for every }\left(y_{1}, \ldots, y_{n}\right) \in \mathcal{X}_{\sigma}\right\}
$$

and

$$
X_{\sigma}=\left\{\left(x_{1}, \ldots, x_{n}\right) \in \mathbb{R}^{n} \mid x_{\sigma(1)}<\cdots<x_{\sigma(n)}\right\} .
$$

Note that expression (3.1) for the signature depends only on the coherent life function $\phi$ and the number of components in the system.

Now, the following theorem yields a representation of the distribution of the system lifetime on the basis of the signature (cf. Navarro et al. (2008c, Theorem 3.6) for weakly exchangeable lifetimes).

Theorem 3.1. Let $X_{1}, \ldots, X_{n}$ be exchangeable lifetimes of components in a coherent system with coherent life function $\phi$. If

$$
\mathrm{P}\left(X_{i}=X_{j}\right)=0, \quad i, j \in\{1, \ldots, n\}, i \neq j,
$$

then the reliability function of the system lifetime $T=\phi\left(X_{1}, \ldots, X_{n}\right)$ is given by

$$
\mathrm{P}(T>t)=\sum_{i=1}^{n} s_{i} \mathrm{P}\left(X_{i: n}>t\right), \quad t \in \mathbb{R},
$$

where $s=\left(s_{1}, \ldots, s_{n}\right)$ denotes the signature of $T$. The entries of $\mathbf{s}$ can be obtained via (3.1). 
The next result shows that the failure-dependent component lifetimes $X_{1}^{(n)}, \ldots, X_{n}^{(n)}$ fulfill the conditions of the previous theorem if the assumptions of Definition 2.1 are satisfied. The mainly technical proof is omitted.

Lemma 3.1. Let the assumptions of Definition 2.1 hold. Then the random variables $X_{1}^{(n)}, \ldots$, $X_{n}^{(n)}$ are exchangeable and they satisfy

$$
\mathrm{P}\left(X_{i}^{(n)}=X_{j}^{(n)}\right)=0, \quad i, j \in\{1, \ldots, n\}, i \neq j .
$$

By combining Theorem 3.1 with the previous result, we obtain a signature-based representation of the reliability function of a system with failure-dependent component lifetimes in terms of the reliability functions of sequential order statistics $X_{*}^{(1)}, \ldots, X_{*}^{(n)}$.

Theorem 3.2. Let the assumptions of Definition 2.1 hold. Let $X_{1}^{(n)}, \ldots, X_{n}^{(n)}$ be the failuredependent lifetimes of the components in a coherent system with coherent life function $\phi$. Then the reliability function of the system lifetime $T^{*}=\phi\left(X_{1}^{(n)}, \ldots, X_{n}^{(n)}\right)$ is given by

$$
\mathrm{P}\left(T^{*}>t\right)=\sum_{i=1}^{n} s_{i} \mathrm{P}\left(X_{*}^{(i)}>t\right), \quad t \in \mathbb{R},
$$

where $s=\left(s_{1}, \ldots, s_{n}\right)$ denotes the signature of $T^{*}$. The entries of $s$ can be obtained via (3.1).

Proof. According to Lemma 3.1, the component lifetimes $X_{1}^{(n)}, \ldots, X_{n}^{(n)}$ satisfy the assumptions of Theorem 3.1. Consequently, the reliability function of the system is a mixture of the reliability functions of the corresponding order statistics $X_{1: n}^{(n)}, \ldots, X_{n: n}^{(n)}$. Because of

$$
\begin{aligned}
\mathrm{P}\left(T^{*}>t\right) & =\sum_{i=1}^{n} s_{i} \mathrm{P}\left(X_{i: n}^{(n)}>t\right) \\
& =\sum_{i=1}^{n} s_{i} \mathrm{P}\left(X_{* *}^{(i)}>t\right) \quad \text { (by Lemma 2.1) } \\
& =\sum_{i=1}^{n} s_{i} \mathrm{P}\left(X_{*}^{(i)}>t\right) \quad \text { (by Theorem 2.1 and Definition 2.1), }
\end{aligned}
$$

the assertion is proved.

By utilizing the last result, the original idea of sequential order statistics, namely to describe the lifetime of a $k$-out-of- $n$ system with failure-dependent component lifetimes, can be extended to lifetimes of general coherent systems. Settings where failure dependence may be reasonably integrated are, for instance, the different types of consecutive $k$-out-of- $n$ systems. A linear consecutive $k$-out-of- $n: F$ system consists of $n$ linearly ordered components which are labeled with the numbers $1, \ldots, n$. Such a system fails if and only if $k$ consecutive components $i, i+1, \ldots, i+k-1$ fail $(1 \leq i \leq i+k-1 \leq n)$. In a circular consecutive $k$-out-of- $n: F$ system the components are arranged in a circle. Consequently, the system fails if and only if $k$ consecutive components in this circle fail. The signatures of both of these consecutive systems are already known. The signatures of linear consecutive $k$-out-of- $n: F$ systems are given in Boland and Samaniego (2004a) (see also Samaniego (2007, p. 64)). Using these results, the signature of a circular consecutive $k$-out-of- $n: F$ system can be obtained by utilizing a relation given in Triantafyllou and Koutras (2008) between the signatures of both systems. Therefore, 
the known signatures can be combined with results for sequential order statistics in order to model consecutive $k$-out-of- $n$ systems in a failure-dependent setting (see Example 4.5 in the next section).

\section{Distribution theory of sequential order statistics}

According to the results of the last section, the distribution theory of sequential order statistics is important in order to obtain the distribution of the lifetime of a coherent system with failuredependent exchangeable component lifetimes. Therefore, we give some results on the joint distribution theory of sequential order statistics based on exchangeable components in this section. Distribution theoretical results for the case of i.i.d. components can be found in Kamps (1995), Cramer and Kamps (2001b), Kamps and Cramer (2001), Cramer and Kamps (2003), and Cramer (2006a).

In the following we assume in general that the random vectors $\left(Y_{1}^{(r)}, \ldots, Y_{n}^{(r)}\right), 1 \leq r \leq n$, satisfy the conditions of Definition 2.1. If they have absolutely continuous distributions, we obtain the following joint density function of sequential order statistics.

Theorem 4.1. For $r \in\{1, \ldots, n\}$, let $\left(Y_{1}^{(r)}, \ldots, Y_{n}^{(r)}\right)$ have a joint density function $f_{r}$ with respect to the n-dimensional Lebesgue measure. Then the density of sequential order statistics is given by

$$
\begin{aligned}
f^{X_{*}^{(1)}, \ldots, X_{*}^{(n)}}\left(x_{1}, \ldots, x_{n}\right) \\
=n ! \int_{x_{1}}^{\infty} \int_{y_{2}}^{\infty} \cdots \int_{y_{n-1}}^{\infty} f_{1}\left(x_{1}, y_{2}, y_{3}, \ldots, y_{n}\right) \mathrm{d} y_{n} \cdots \mathrm{d} y_{3} \mathrm{~d} y_{2} \\
\quad \times \prod_{r=1}^{n-1} \frac{\int_{x_{r+1}}^{\infty} \int_{y_{r+2}}^{\infty} \cdots \int_{y_{n-1}}^{\infty} f_{r+1}\left(x_{1}, \ldots, x_{r+1}, y_{r+2}, y_{r+3}, \ldots, y_{n}\right) \mathrm{d} y_{n} \cdots \mathrm{d} y_{r+3} \mathrm{~d} y_{r+2}}{\int_{x_{r}}^{\infty} \int_{y_{r+1}}^{\infty} \cdots \int_{y_{n-1}}^{\infty} f_{r+1}\left(x_{1}, \ldots, x_{r}, y_{r+1}, y_{r+2}, \ldots, y_{n}\right) \mathrm{d} y_{n} \cdots \mathrm{d} y_{r+2} \mathrm{~d} y_{r+1}}
\end{aligned}
$$

for $x_{1} \leq \cdots \leq x_{n}$. Alternatively, the density function can be rewritten as

$$
\begin{aligned}
f^{X_{*}^{(1)}, \ldots, X_{*}^{(n)}}\left(x_{1}, \ldots, x_{n}\right) \\
=n ! \int_{x_{1}}^{\infty} \int_{x_{1}}^{y_{n}} \cdots \int_{x_{1}}^{y_{3}} f_{1}\left(x_{1}, y_{2}, \ldots, y_{n-1}, y_{n}\right) \mathrm{d} y_{2} \cdots \mathrm{d} y_{n-1} \mathrm{~d} y_{n} \\
\quad \times \prod_{r=1}^{n-1} \frac{\int_{x_{r+1}}^{\infty} \int_{x_{r+1}}^{y_{n}} \cdots \int_{x_{r+1}}^{y_{r+3}} f_{r+1}\left(x_{1}, \ldots, x_{r+1}, y_{r+2}, \ldots, y_{n-1}, y_{n}\right) \mathrm{d} y_{r+2} \cdots \mathrm{d} y_{n-1} \mathrm{~d} y_{n}}{\int_{x_{r}}^{\infty} \int_{x_{r}}^{y_{n}} \cdots \int_{x_{r}}^{y_{r+2}} f_{r+1}\left(x_{1}, \ldots, x_{r}, y_{r+1}, \ldots, y_{n-1}, y_{n}\right) \mathrm{d} y_{r+1} \cdots \mathrm{d} y_{n-1} \mathrm{~d} y_{n}}
\end{aligned}
$$

for $x_{1} \leq \cdots \leq x_{n}$.

Proof. Since $Y_{1}^{(r)}, \ldots, Y_{n}^{(r)}$ are exchangeable, the density of $Y_{1: n}^{(r)}, \ldots, Y_{n: n}^{(r)}$ is given by

$$
f^{Y_{1: n}^{(r)}, \ldots, Y_{n: n}^{(r)}}\left(x_{1}, \ldots, x_{n}\right)=n ! f_{r}\left(x_{1}, \ldots, x_{n}\right), \quad x_{1} \leq \cdots \leq x_{n} .
$$

Consequently,

$$
f^{X_{*}^{(1)}}\left(x_{1}\right)=n ! \int_{x_{1}}^{\infty} \int_{y_{2}}^{\infty} \cdots \int_{y_{n-1}}^{\infty} f_{1}\left(x_{1}, y_{2}, y_{3}, \ldots, y_{n}\right) \mathrm{d} y_{n} \cdots \mathrm{d} y_{3} \mathrm{~d} y_{2}
$$


and, moreover,

$$
\begin{aligned}
& f^{X_{*}^{(r+1)} \mid X_{*}^{(r)}, \ldots, X_{*}^{(1)}}\left(x_{r+1} \mid x_{r}, \ldots, x_{1}\right) \\
& =f^{Y_{r+1: n}^{(r+1)} \mid Y_{r: n}^{(r+1)}, \ldots, Y_{1: n}^{(r+1)}}\left(x_{r+1} \mid x_{r}, \ldots, x_{1}\right) \\
& =\frac{f^{Y_{1: n}^{(r+1)}, \ldots, Y_{r: n}^{(r+1)}, Y_{r+1: n}^{(r+1)}}\left(x_{1}, \ldots, x_{r}, x_{r+1}\right)}{f^{Y_{1: n}^{(r+1)}, \ldots, Y_{r: n}^{(r+1)}}\left(x_{1}, \ldots, x_{r}\right)} \\
& =\frac{\int_{x_{r+1}}^{\infty} \int_{y_{r+2}}^{\infty} \cdots \int_{y_{n-1}}^{\infty} f_{r+1}\left(x_{1}, \ldots, x_{r+1}, y_{r+2}, y_{r+3}, \ldots, y_{n}\right) \mathrm{d} y_{n} \cdots \mathrm{d} y_{r+3} \mathrm{~d} y_{r+2}}{\int_{x_{r}}^{\infty} \int_{y_{r+1}}^{\infty} \cdots \int_{y_{n-1}}^{\infty} f_{r+1}\left(x_{1}, \ldots, x_{r}, y_{r+1}, y_{r+2}, \ldots, y_{n}\right) \mathrm{d} y_{n} \cdots \mathrm{d} y_{r+2} \mathrm{~d} y_{r+1}}
\end{aligned}
$$

for $x_{r+1} \geq x_{r}$, which leads to the given result. The second representation is obtained by changing the order of integration.

Remark 4.1. If $f_{1}=f_{2}=\cdots=f_{n}=f$, sequential order statistics $X_{*}^{(1)}, \ldots, X_{*}^{(n)}$ coincide with order statistics $X_{1: n}, \ldots, X_{n: n}$ from exchangeable random variables with density $f$, i.e.

$$
f^{X_{1: n}, \ldots, X_{n: n}}\left(x_{1}, \ldots, x_{n}\right)=n ! f\left(x_{1}, \ldots, x_{n}\right), \quad x_{1} \leq \cdots \leq x_{n} .
$$

If the exchangeable random variables are generated by mixing conditionally i.i.d. random variables, then the joint density of sequential order statistics simplifies. The resulting representation is given in the following corollary.

Corollary 4.1. Let $\Theta_{1}, \ldots, \Theta_{n}$ be random variables with distribution functions $G_{1}, \ldots, G_{n}$, respectively. For $r \in\{1, \ldots, n\}$, let $\left(Y_{1}^{(r)}, \ldots, Y_{n}^{(r)}\right)$ have a joint density function

$$
f_{r}\left(y_{1}, \ldots, y_{n}\right)=\int \prod_{i=1}^{n} f_{r}\left(y_{i} \mid \theta\right) \mathrm{d} G_{r}(\theta),
$$

where $f_{r}(\cdot \mid \theta)$ denotes a density with respect to the Lebesgue measure for every $\theta$ in the support of $\Theta_{r}$. Then the joint density function of sequential order statistics is given by

$$
\begin{aligned}
f^{X_{*}^{(1)}, \ldots, X_{*}^{(n)}}\left(x_{1}, \ldots, x_{n}\right) \\
=n ! \int f_{1}\left(x_{1} \mid \theta\right)\left(\bar{F}_{1}\left(x_{1} \mid \theta\right)\right)^{n-1} \mathrm{~d} G_{1}(\theta) \\
\quad \times \prod_{r=1}^{n-1} \frac{\int\left(\prod_{i=1}^{r+1} f_{r+1}\left(x_{i} \mid \theta\right)\right)\left(\bar{F}_{r+1}\left(x_{r+1} \mid \theta\right)\right)^{n-r-1} \mathrm{~d} G_{r+1}(\theta)}{\int\left(\prod_{i=1}^{r} f_{r+1}\left(x_{i} \mid \theta\right)\right)\left(\bar{F}_{r+1}\left(x_{r} \mid \theta\right)\right)^{n-r} \mathrm{~d} G_{r+1}(\theta)}, \quad x_{1} \leq \cdots \leq x_{n},
\end{aligned}
$$

where $F_{r}(\cdot \mid \theta)$ denotes the distribution function with density $f_{r}(\cdot \mid \theta)(1 \leq r \leq n)$.

Proof. Since the marginal density of order statistics from these particular exchangeable random variables is given by

$$
\begin{aligned}
& f_{1: n}^{Y_{1: n}^{(r)}, \ldots, Y_{r: n}^{(r)}}\left(x_{1}, \ldots, x_{r}\right) \\
& \quad=\frac{n !}{(n-r) !} \int\left(\prod_{i=1}^{r} f_{r}\left(x_{i} \mid \theta\right)\right)\left(\bar{F}_{r}\left(x_{r} \mid \theta\right)\right)^{n-r} \mathrm{~d} G_{r}(\theta),
\end{aligned}
$$

the result follows. 
In the remainder we treat a generalization of the model considered in Example 2.1 in detail. In the following model, sequential order statistics based on independent, identically exponentially distributed random variables with different rate parameters (cf. Remark 2.3 and Remark 4.2) appear as a limit case. Let $\alpha_{1} \ldots, \alpha_{n}>0$. We define

$$
\left(Y_{1}^{(r)}, \ldots, Y_{n}^{(r)}\right)=\left(\frac{X_{1}}{\alpha_{r} V_{r}}, \ldots, \frac{X_{n}}{\alpha_{r} V_{r}}\right), \quad 1 \leq r \leq n,
$$

where $X_{1}, \ldots, X_{n}$ are i.i.d. random variables that are distributed according to a standard exponential distribution. The random variable $V_{r}$ is independent of $X_{1}, \ldots, X_{n}$ and follows a gamma distribution with shape parameter $a_{r}>0$ and scale parameter $1 / a_{r}$ (in particular, $\mathrm{E}\left(V_{r}\right)=1$ and $\left.\operatorname{var}\left(V_{r}\right)=1 / a_{r}\right)$. This setting fulfills the conditions of Corollary 4.1 by setting, e.g. $\Theta_{r}=V_{r}$ and

$$
f_{r}(x \mid \theta)=\alpha_{r} \theta \exp \left[-\alpha_{r} \theta x\right], \quad x>0,
$$

for every $r \in\{1, \ldots, n\}$. The resulting joint distribution of $Y_{1}^{(r)}, \ldots, Y_{n}^{(r)}$ has the density function

$$
f_{r}\left(y_{1}, \ldots, y_{n}\right)=\frac{\Gamma\left(a_{r}+n\right)}{\Gamma\left(a_{r}\right)}\left(\frac{\alpha_{r}}{a_{r}}\right)^{n}\left(1+\frac{\alpha_{r}}{a_{r}} \sum_{i=1}^{n} y_{i}\right)^{-\left(a_{r}+n\right)}, \quad y_{1}, \ldots, y_{n}>0 .
$$

It is well known that this is a multivariate Pareto distribution of the second kind (cf. Johnson et al. (2000, pp. 602-605)) with

$$
\begin{gathered}
\mathrm{E}\left(Y_{1}^{(r)}\right)=\frac{a_{r}}{\alpha_{r}\left(a_{r}-1\right)}, \quad a_{r}>1, \quad \operatorname{var}\left(Y_{1}^{(r)}\right)=\frac{a_{r}^{3}}{\alpha_{r}^{2}\left(a_{r}-2\right)\left(a_{r}-1\right)^{2}}, \quad a_{r}>2, \\
\operatorname{cov}\left(Y_{1}^{(r)}, Y_{2}^{(r)}\right)=\frac{a_{r}^{2}}{\alpha_{r}^{2}\left(a_{r}-2\right)\left(a_{r}-1\right)^{2}}, \quad a_{r}>2 .
\end{gathered}
$$

Since $V_{r}$ converges in distribution to the constant 1 if $a_{r} \rightarrow \infty$, it follows from (4.1) and the continuous mapping theorem that in this case $Y_{1}^{(r)}, \ldots, Y_{n}^{(r)}$ converge in distribution to independent, identically exponentially distributed random variables with scale parameter $1 / \alpha_{r}$.

By applying Corollary 4.1, it can be shown that the joint density of sequential order statistics based on (4.1) is given by (with $0=: x_{0} \leq x_{1} \leq \cdots \leq x_{n}$ )

$$
\begin{aligned}
f^{X_{*}^{(1)}, \ldots, X_{*}^{(n)}}\left(x_{1}, \ldots, x_{n}\right) \\
=n !\left(\prod_{j=1}^{n} \frac{\alpha_{j}\left(a_{j}+j-1\right)}{a_{j}}\right) \\
\quad \times \prod_{j=1}^{n} \frac{\left(1+\left(\alpha_{j} / a_{j}\right)\left(\sum_{i=1}^{j-1} x_{i}+(n-j+1) x_{j}\right)\right)^{-\left(a_{j}+j\right)}}{\left(1+\left(\alpha_{j} / a_{j}\right)\left(\sum_{i=1}^{j-2} x_{i}+(n-j+2) x_{j-1}\right)\right)^{-\left(a_{j}+j-1\right)}} .
\end{aligned}
$$

For appropriately normalized spacings from these sequential order statistics, the following relation holds. A well-known result for these spacings in the situation of Remark 2.3 with an underlying standard exponential distribution $F$ is included by passing to the limit (see Remark 4.2, below). 
Theorem 4.2. Let $X_{*}^{(1)}, \ldots, X_{*}^{(n)}$ be sequential order statistics based on the random vectors (4.1). Then the distribution of

$$
D_{*}^{(r)}=(n-r+1) \alpha_{r}\left(X_{*}^{(r)}-X_{*}^{(r-1)}\right), \quad 1 \leq r \leq n
$$

$\left(\right.$ with $\left.X_{*}^{(0)}:=0\right)$, satisfies the relation

$$
D_{*}^{(r)} \sim Z_{r}\left(1+\frac{\alpha_{r}}{a_{r}} \sum_{k=1}^{r-1} \frac{D_{*}^{(k)}}{\alpha_{k}}\right), \quad 1 \leq r \leq n,
$$

with independent random variables $Z_{1}, \ldots, Z_{n}$, where $Z_{r}$ follows a Pareto type II distribution with scale parameter $a_{r}$ and shape parameter $a_{r}+r-1$, i.e. the distribution function of $Z_{r}$ is given by

$$
F^{Z_{r}}(t)=1-\left(1+\frac{t}{a_{r}}\right)^{-\left(a_{r}+r-1\right)}, \quad t \geq 0 .
$$

Proof. The density of $D_{*}^{(1)}, \ldots, D_{*}^{(n)}$ is given by

$$
\begin{aligned}
f^{D_{*}^{(1)}, \ldots,} D_{*}^{(n)}\left(d_{1}, \ldots, d_{n}\right) \\
=\prod_{j=1}^{n} \frac{a_{j}+j-1}{a_{j}} \\
\quad \times \prod_{j=1}^{n} \frac{\left(1+\left(\alpha_{j} / a_{j}\right)\left(\sum_{k=1}^{j} d_{k} / \alpha_{k}\right)\right)^{-\left(a_{j}+j\right)}}{\left(1+\left(\alpha_{j} / a_{j}\right)\left(\sum_{k=1}^{j-1} d_{k} / \alpha_{k}\right)\right)^{-\left(a_{j}+j-1\right)}}, \quad d_{1}, \ldots, d_{n} \geq 0 .
\end{aligned}
$$

It can be shown that

$$
\begin{aligned}
& f^{D_{*}^{(r)} \mid D_{*}^{(1)}, \ldots, D_{*}^{(r-1)}}\left(d_{r} \mid d_{1}, \ldots, d_{r-1}\right) a_{r}+r-1 \\
& \quad=\frac{a_{r}}{a_{r}+\alpha_{r} \sum_{k=1}^{r-1} d_{k}}\left(1+\frac{d_{r}}{a_{r}+\alpha_{r} \sum_{k=1}^{r-1} d_{k} / \alpha_{k}}\right)^{-\left(a_{r}+r\right)}, \quad d_{r} \geq 0 .
\end{aligned}
$$

Consequently, $D_{*}^{(r)} \mid D_{*}^{(1)}=d_{1}, \ldots, D_{*}^{(r-1)}=d_{r-1}$ follows a Pareto type II distribution with scale parameter $a_{r}+\alpha_{r} \sum_{k=1}^{r-1} d_{k} / \alpha_{k}$ and shape parameter $a_{r}+r-1$. Thus, we obtain the relation

$$
D_{*}^{(r)} \sim \widetilde{Z}_{r}\left(a_{r}+\alpha_{r} \sum_{k=1}^{r-1} \frac{D_{*}^{(k)}}{\alpha_{k}}\right), \quad 1 \leq r \leq n,
$$

with independent random variables $\widetilde{Z}_{1}, \ldots, \widetilde{Z}_{n}$, where $\widetilde{Z}_{r}$ follows a Pareto type II distribution with scale parameter 1 and shape parameter $a_{r}+r-1$. By rewriting the relation with $Z_{r}=a_{r} \widetilde{Z}_{r}$, we arrive at the claimed result.

Remark 4.2. In the case considered in Remark 2.3, if $F$ is chosen as a standard exponential distribution then the CDFs $F_{r}$ follow exponential distributions with possibly different rate parameters, i.e.

$$
F_{r}(t)=1-\mathrm{e}^{-\alpha_{r} t}, \quad t \geq 0,1 \leq r \leq n .
$$

It is well known that in this case the spacings

$$
D_{*}^{(r)}=(n-r+1) \alpha_{r}\left(X_{*}^{(r)}-X_{*}^{(r-1)}\right), \quad 1 \leq r \leq n,
$$


are i.i.d. and they are again distributed according to $F$ (cf. Kamps $(1995$, p. 81)). This result can be obtained from Theorem 4.2 via a limit approach. Let $a_{1}=\cdots=a_{n}=a$. Note that $Z_{r}$ converges in distribution to a standard exponentially distributed random variable if $a \rightarrow \infty$. Hence, the claimed result follows from the relation in Theorem 4.2 and the continuous mapping theorem by letting $a \rightarrow \infty$. Moreover, Theorem 4.2 can be regarded as a generalization of this particular result.

Remark 4.3. Since $\mathrm{E}\left(Z_{1}\right)=a_{1} /\left(a_{1}-1\right)$ (if $\left.a_{1}>1\right)$ and $\mathrm{E}\left(Z_{r}\right)=a_{r} /\left(a_{r}+r-2\right), 2 \leq r \leq n$, Theorem 4.2 yields the recurrence relation

$$
\mathrm{E}\left(D_{*}^{(r)}\right)=\frac{a_{r}}{a_{r}+r-2}+\frac{\alpha_{r}}{a_{r}+r-2} \sum_{k=1}^{r-1} \frac{\mathrm{E}\left(D_{*}^{(k)}\right)}{\alpha_{k}}
$$

with

$$
\mathrm{E}\left(D_{*}^{(1)}\right)=\frac{a_{1}}{a_{1}-1} \quad \text { if } a_{1}>1 .
$$

In particular, no assumptions on $a_{2}, \ldots, a_{n}$ are needed. In the case in which $a_{1}=\cdots=a_{n}=$ $a>1$, the expressions can be further simplified. It can be shown by induction that

$$
\sum_{k=1}^{r} \frac{\mathrm{E}\left(D_{*}^{(k)}\right)}{\alpha_{k}}=a(a+r-1) \sum_{k=1}^{r} \frac{1}{\alpha_{k}(a+k-2)(a+k-1)},
$$

which in turn yields

$$
\begin{aligned}
\mathrm{E}\left(D_{*}^{(r)}\right) & =\frac{a}{a+r-2}+a \alpha_{r} \sum_{k=1}^{r-1} \frac{1}{\alpha_{k}(a+k-2)(a+k-1)} \\
& =\frac{a}{a+r-1}+a \alpha_{r} \sum_{k=1}^{r} \frac{1}{\alpha_{k}(a+k-2)(a+k-1)} .
\end{aligned}
$$

Finally, we apply the previous results in order to calculate the mean system lifetime of some concrete coherent systems in the setting of model (4.1).

Remark 4.4. For the two-component parallel system in Example 2.1, we obtain (if $a>1$ )

$$
\mathrm{E}\left(D_{*}^{(1)}\right)=\frac{a}{a-1}, \quad \mathrm{E}\left(D_{*}^{(2)}\right)=1+\frac{\alpha_{2}}{\alpha_{1}(a-1)},
$$

and, consequently, the mean of the lifetime $T_{2: 2}^{*}=X_{*}^{(2)}$ of the two-component parallel system with failure-dependent component lifetimes is given by

$$
\mathrm{E}\left(X_{*}^{(2)}\right)=\frac{\mathrm{E}\left(D_{*}^{(2)}\right)}{\alpha_{2}}+\frac{\mathrm{E}\left(D_{*}^{(1)}\right)}{2 \alpha_{1}}=\frac{1}{\alpha_{2}}+\frac{a+2}{2 \alpha_{1}(a-1)} .
$$

Remark 4.5. By utilizing Theorem 3.2, we can find the mean system lifetimes of consecutive $k$-out-of- $n: F$ systems. We give two examples.

- The signature $\boldsymbol{s}=\left(s_{1}, s_{2}, s_{3}\right)$ of a linear consecutive 2-out-of-3: $F$ system is given by (cf. Samaniego (2007, p. 64))

$$
s_{1}=0, \quad s_{2}=\frac{2}{3}, \quad s_{3}=\frac{1}{3} .
$$


By applying Theorem 3.2, the mean of the lifetime $T_{c: 2: 3}^{*}$ of the linear consecutive 2-outof-3: $F$ system with failure-dependent component lifetimes is given by

$$
\mathrm{E}\left(T_{c: 2: 3}^{*}\right)=\frac{2}{3} \mathrm{E}\left(X_{*}^{(2)}\right)+\frac{1}{3} \mathrm{E}\left(X_{*}^{(3)}\right) .
$$

If the sequential order statistics are based on the random vectors (4.1) with $a_{1}=a_{2}=$ $a_{3}=a>1$, we obtain

$$
\begin{aligned}
\mathrm{E}\left(X_{*}^{(2)}\right) & =\frac{\mathrm{E}\left(D_{*}^{(2)}\right)}{2 \alpha_{2}}+\frac{\mathrm{E}\left(D_{*}^{(1)}\right)}{3 \alpha_{1}}=\frac{1}{2 \alpha_{2}}+\frac{2 a+3}{6 \alpha_{1}(a-1)} \\
\mathrm{E}\left(X_{*}^{(3)}\right) & =\frac{\mathrm{E}\left(D_{*}^{(3)}\right)}{\alpha_{3}}+\frac{\mathrm{E}\left(D_{*}^{(2)}\right)}{2 \alpha_{2}}+\frac{\mathrm{E}\left(D_{*}^{(1)}\right)}{3 \alpha_{1}} \\
& =\frac{a}{\alpha_{3}(a+1)}+\frac{a+3}{2 \alpha_{2}(a+1)}+\frac{2 a+9}{6 \alpha_{1}(a-1)} .
\end{aligned}
$$

Hence, the mean of the lifetime $T_{c: 2: 3}^{*}$ has the explicit form

$$
\mathrm{E}\left(T_{c: 2: 3}^{*}\right)=\frac{1}{3}\left(\frac{a}{\alpha_{3}(a+1)}+\frac{3 a+5}{2 \alpha_{2}(a+1)}+\frac{2 a+5}{2 \alpha_{1}(a-1)}\right) .
$$

- It can be shown (cf. Triantafyllou and Koutras (2008)) that the signature $s=\left(s_{1}, s_{2}, s_{3}, s_{4}\right)$ of a circular consecutive 2-out-of-4: $F$ system is given by

$$
s_{1}=0, \quad s_{2}=\frac{2}{3}, \quad s_{3}=\frac{1}{3}, \quad s_{4}=0 .
$$

Thus, the mean of the lifetime $T_{c c: 2: 4}^{*}$ of the circular consecutive 2-out-of-4: $F$ system with failure-dependent component lifetimes is also given by

$$
\mathrm{E}\left(T_{c c: 2: 4}^{*}\right)=\frac{2}{3} \mathrm{E}\left(X_{*}^{(2)}\right)+\frac{1}{3} \mathrm{E}\left(X_{*}^{(3)}\right) .
$$

However, note that the occurring sequential order statistics depend on $n=4$. If model (4.1) with $a_{1}=\cdots=a_{4}=a>1$ is considered, we obtain the expectations

$$
\begin{aligned}
\mathrm{E}\left(X_{*}^{(2)}\right) & =\frac{\mathrm{E}\left(D_{*}^{(2)}\right)}{3 \alpha_{2}}+\frac{\mathrm{E}\left(D_{*}^{(1)}\right)}{4 \alpha_{1}}=\frac{1}{3 \alpha_{2}}+\frac{3 a+4}{12 \alpha_{1}(a-1)}, \\
\mathrm{E}\left(X_{*}^{(3)}\right) & =\frac{\mathrm{E}\left(D_{*}^{(3)}\right)}{2 \alpha_{3}}+\frac{\mathrm{E}\left(D_{*}^{(2)}\right)}{3 \alpha_{2}}+\frac{\mathrm{E}\left(D_{*}^{(1)}\right)}{4 \alpha_{1}} \\
& =\frac{a}{2 \alpha_{3}(a+1)}+\frac{2 a+5}{6 \alpha_{2}(a+1)}+\frac{3 a+10}{12 \alpha_{1}(a-1)} .
\end{aligned}
$$

The mean of the lifetime $T_{c c: 2: 4}^{*}$ is therefore given by

$$
\mathrm{E}\left(T_{c c: 2: 4}^{*}\right)=\frac{1}{3}\left(\frac{a}{2 \alpha_{3}(a+1)}+\frac{2 a+3}{2 \alpha_{2}(a+1)}+\frac{3 a+6}{4 \alpha_{1}(a-1)}\right) .
$$

In particular, we have $\mathrm{E}\left(T_{c c: 2: 4}^{*}\right)<\mathrm{E}\left(T_{c: 2: 3}^{*}\right)$ for every choice of $\alpha_{1}, \alpha_{2}, \alpha_{3}>0$.

\section{Acknowledgement}

The author would like to thank an anonymous referee for several helpful comments. 


\section{References}

Balakrishnan, N., Beutner, E. AND Kamps, U. (2008). Order restricted inference for sequential $k$-out-of- $n$ systems. J. Multivariate Anal. 99, 1489-1502.

Barlow, R. E. AND Proschan, F. (1975). Statistical Theory of Reliability and Life Testing. Holt, Rinehart and Winston, New York.

BeUtner, E. (2008). Nonparametric inference for sequential $k$-out-of- $n$ systems. Ann. Inst. Statist. Math. 60, $605-626$.

Beutner, E., Burkschat, M. And Kamps, U. (2007). Sequential $k$-out-of- $n$ systems: model and estimation. In Proc. 5th Internat. Math. Meth. Reliab. Conf., Glasgow.

Boland, P. J. AND Samaniego, F. J. (2004a). Stochastic ordering results for consecutive $k$-out-of- $n: F$ systems. IEEE Trans. Reliab. 53, 7-10.

Boland, P. J. AND SAmaniego, F. (2004b). The signature of a coherent system and its applications in reliability. In Mathematical Reliability: An Expository Perspective, eds R. Soyer, T. Mazzuchi and N. D. Singpurwalla, Kluwer, Boston, MA, pp. 1-29.

Boland, P. J., Samaniego, F. and Vestrup, E. M. (2003). Linking dominations and signatures in network reliability theory. In Mathematical and Statistical Methods in Reliability, eds B. Lindquist and K. A. Doksum, World Scientific, Singapore, pp. 89-103.

CRAMER, E. (2001). Inference for stress-strength models based on Weinman multivariate exponential samples. Commun. Statist. Theory Methods 30, 331-346.

Cramer, E. (2006a). Dependence structure of generalized order statistics. Statistics 40, 409-413.

Cramer, E. (2006b). Sequential order statistics. In Encyclopedia of Statistical Sciences, Vol. 12, 2nd edn. John Wiley, Hoboken, NJ, pp. 7629-7634.

Cramer, E. And Kamps, U. (1996). Sequential order statistics and $k$-out-of- $n$ systems with sequentially adjusted failure rates. Ann. Inst. Statist. Math. 48, 535-549.

CRAmer, E. AND KAmps, U. (1998). Sequential $k$-out-of- $n$ systems with Weibull components. Econom. Quality Control 13, 227-239.

Cramer, E. and Kamps, U. (2001a). Estimation with sequential order statistics from exponential distributions. Ann. Inst. Statist. Math. 53, 307-324.

Cramer, E. And Kamps, U. (2001b). Sequential $k$-out-of- $n$ systems. In Advances in Reliability (Handbook Statist. 20), eds N. Balakrishnan and C. R. Rao, North-Holland, Amsterdam, pp. 301-372.

Cramer, E. and Kamps, U. (2003). Marginal distributions of sequential and generalized order statistics. Metrika 58, 293-310.

David, H. A. And Nagaraja, H. N. (2003). Order Statistics, 3rd edn. John Wiley, Hoboken, NJ.

Esary, J. D. and Marshall, A. W. (1970). Coherent life functions. SIAM J. Appl. Math. 18, 810-814.

Garren, S. T. and Richards, D. S. P. (1998). General conditions for comparing the reliability functions of systems of components sharing a common environment. J. Appl. Prob. 35, 124-135.

Gupta, R. C. (2002). Reliability of a $k$-out-of- $n$ system of components sharing a common environment. Appl. Math. Lett. 15, 837-844.

HáJeK, J., ŠIDÁK, Z. AND Sen, P. K. (1999). Theory of Rank Tests, 2nd edn. Academic Press, San Diego, CA.

KAMPS, U. (1995). A Concept of Generalized Order Statistics. Teubner, Stuttgart.

KAMPS, U. AND CRAMER, E. (2001). On distributions of generalized order statistics. Statistics 35, 269-280.

Khaledi, B.-E. And Shaked, M. (2007). Ordering conditional lifetimes of coherent systems. J. Statist. Planning Infer. 137, 1173-1184.

Kochar, S., Mukerjee, H. and Samaniego, F. J. (1999). The 'signature' of a coherent system and its application to comparisons among systems. Naval Res. Logistics 46, 507-523.

Kotz, S., Balakrishnan, N. And Johnson, N. L. (2000). Continuous Multivariate Distributions, Vol. 1, 2nd edn. Wiley-Interscience, New York.

Lindley, D. V. AND SingPurwalla, N. D. (1986). Multivariate distributions for the life lengths of components of a system sharing a comment environment. J. Appl. Prob. 23, 418-431.

Navarro, J. and Eryilmaz, S. (2007). Mean residual lifetimes of consecutive $k$-out-of- $n$ systems. J. Appl. Prob. 44, 82-98.

Navarro, J. And Hernandez, P. J. (2008). Mean residual life functions of finite mixtures, order statistics and coherent systems. Metrika 67, 277-298.

NAVARro, J. AND RyChlik, T. (2007). Reliability and expectation bounds for coherent systems with exchangeable components. J. Multivariate Anal. 98, 102-113.

Navarro, J. and Shaked, M. (2006). Hazard rate ordering of order statistics and systems. J. Appl. Prob. 43, $391-408$.

Navarro, J., Balakrishnan, N. And Samaniego, F. J. (2008a). Mixture representations of residual lifetimes of used systems. J. Appl. Prob. 45, 1097-1112.

Navarro, J., Ruiz, J. M. and Sandoval, C. J. (2005). A note on comparisons among coherent systems with dependent components using signatures. Statist. Prob. Lett. 72, 179-185. 
Navarro, J., Ruiz, J. M. and Sandoval, C. J. (2007a). Properties of coherent systems with dependent components. Commun. Statist. Theory Meth. 36, 175-191.

Navarro, J., Ruiz, J. M. and Sandoval, C. J. (2008b). Properties of systems with two exchangeable Pareto components. Statist. Papers 49, 177-190.

Navarro, J., Rychlik, T. and ShaKed, M. (2007b). Are the order statistics ordered? A survey of recent results. Commun. Statist. Theory Meth. 36, 1273-1290.

Navarro, J., Samaniego, F. J., Balakrishnan, N. and Bhattacharya, D. (2008c). On the application and extension of system signatures in engineering reliability. Naval Res. Logistics 55, 313-327.

Revathy, S. A. And Chandrasekar, B. (2007). Equivariant estimation of parameters based on sequential order statistics from $(1,3)$ and $(2,3)$ systems. Commun. Statist. Theory Meth. 36, 541-548.

Samaniego, F. J. (1985). On closure of the IFR class under formation of coherent systems. IEEE Trans. Reliab. 34, 69-72.

Samaniego, F. J. (2007). System Signatures and Their Applications in Engineering Reliability. Springer, New York.

Triantafyllou, I. S. and Koutras, M. V. (2008). On the signature of coherent systems and applications. Prob. Eng. Inf. Sci. 22, 19-35.

Zhuang, W. And Hu, T. (2007). Multivariate stochastic comparisons of sequential order statistics. Prob. Eng. Inf. Sci. 21, 47-66. 